\title{
Intermediate-risk grouping of cervical cancer patients treated with radical hysterectomy: a Korean Gynecologic Oncology Group study
}

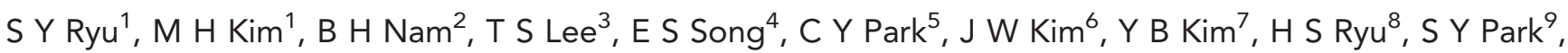

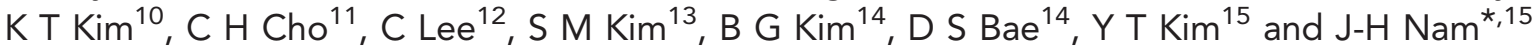

${ }^{1}$ Department of Obstetrics and Gynecology, Korea Cancer Center Hospital, Korea Institute of Radiological and Medical Sciences, 75 Nowon-gil, Nowon-gu, Seoul 139-706, Korea; ${ }^{2}$ Biometric Research Branch, Research Institute and Hospital, National Cancer Center, 323 IIsan-ro, Ilsandong-gu, Goyang, Gyeonggi-do 410-769, Korea; ${ }^{3}$ Department of Obstetrics and Gynecology, SMG-SNU Boramae Medical Center, 20 Boramae-ro 5-gil, Dongjak-gu, Seoul 156-707, Korea; ${ }^{4}$ Department of Obstetrics and Gynecology, Inha University School of Medicine, 7-206 Sinheung-dong, Jung-gu, Incheon 400-711, Korea; ${ }^{5}$ Department of Obstetrics and Gynecology, Gachon University Gil Medical Center, 1198 Guwol-dong, Namdong-gu, Incheon 405-760, Korea; ${ }^{6}$ Department of Obstetrics and Gynecology, College of Medicine, Seoul National University, 101 Daehak-ro, Jongno-gu, Seoul 110-744, Korea; 'Department of Obstetrics and Gynecology, Seoul National University Bundang Hospital, 82 Gumi-ro 173 Beon-gil, Bundang-gu, Seoungnam 463-707, Korea; ${ }^{8}$ Department of Obstetrics and Gynecology, Ajou University School of Medicine, San 5, Wonchon-dong, Yeongtong-gu, Suwon 443-721, Korea; ${ }^{9}$ Center for Uterine Cancer, Research Institute and Hospital, National Cancer Center, 323 Ilsan-ro, Ilsandong-gu, Goyang 410-769, Korea; ${ }^{10}$ Department of Obstetrics and Gynecology, Busan Paik Hospital, Inje University, Gaegum-dong 633-165, Busanjin-gu, Busan 614-735, Korea; ${ }^{11}$ Department of Obstetrics and Gynecology, Dongsan Medical Center, Keimyung University, 56 Dalseong-ro, Jung-gu, Daegu 700-712, Korea; ${ }^{12}$ Department of Obstetrics and Gynecology, Sanggye Paik Hospital, Inje University College of Medicine, 1342 Dongil-ro, Nowon-gu, Seoul 139-707, Korea; ${ }^{13}$ Department of Obstetrics and Gynecology, Chonnam National University Medical School, 42 Jebong-ro, Dong-gu, Gwangju 501-757, Korea; ${ }^{14}$ Department of Obstetrics and Gynecology, Samsung Medical Center, Sungkyunkwan University School of Medicine, 81 Irwon-ro Gangnam-gu, Seoul 135-710, Korea and ${ }^{15}$ Department of Obstetrics and Gynecology, University of Ulsan College of Medicine, Asan Medical Center, 88 Olympic-ro 43-Gil, Songpa-gu, Seoul 138-736, Korea

Background: In this study, we sought to identify a criterion for the intermediate-risk grouping of patients with cervical cancer who exhibit any intermediate-risk factor after radical hysterectomy.

Methods: In total, 2158 patients with pathologically proven stage IB-IIA cervical cancer with any intermediate-risk factor after radical hysterectomy were randomly assigned to two groups, a development group and a validation group, at a ratio of 3:1 (1620 patients:538 patients). To predict recurrence, multivariate models were developed using the development group. The ability of the models to discriminate between groups was validated using the log-rank test and receiver operating characteristic (ROC) analysis.

Results: Four factors (histology, tumour size, deep stromal invasion (DSI), and lymphovascular space involvement (LVSI)) were significantly associated with disease recurrence and included in the models. Among the nine possible combinations of the four variables, models consisting of any two of the four intermediate-risk factors (tumour size $\geqslant 3 \mathrm{~cm}$, DSI of the outer third of the cervix, LVSI, and adenocarcinoma or adenosquamous carcinoma histology) demonstrated the best performance for predicting recurrence.

Conclusion: This study identified a 'four-factor model' in which the presence of any two factors may be useful for predicting recurrence in patients with cervical cancer treated with radical hysterectomy.

*Correspondence: Professor J-H Nam; E-mail: jhnam@amc.seoul.kr

The abstract was submitted as an oral presentation at the IGCS meeting in Vancouver, Canada, 2012.

Received 5 August 2013; revised 16 October 2013; accepted 21 October 2013; published online 19 December 2013

(c) 2014 Cancer Research UK. All rights reserved 0007-0920/14 
Most patients with early cervical cancer are treated by radical hysterectomy followed by adjuvant chemoradiation, according to the presence of clinicopathologic risk factors (Delgado et al, 1989, 1990; Sedlis et al, 1999; Peters et al, 2000; Van de Putte et al, 2005; Park et al, 2010a; Ryu et al, 2011). Because patients with early cervical cancer have a favourable prognosis and adjuvant radiation treatment is associated with considerable acute or chronic adverse effects, identification of this high-risk group is crucial for physicians to avoid overtreatment (Sedlis et al, 1999; Peters et al, 2000; Ryu et al, 2011). Several clinicopathologic factors, such as tumour size, histology, lymphovascular space tumour involvement (LVSI), lymph node metastasis, and parametrial involvement, have been identified and analysed for prognostic significance (Delgado et al, 1989; Fuller et al, 1989; Samlal et al, 1997).

Among these variables, several risk factors including positive lymph nodes, parametrial tumour invasion, and a positive resection margin were defined as high-risk factors because they were shown to increase the recurrence rate to $25-30 \%$, necessitating adjuvant chemoradiation treatment (Delgado et al, 1989; Fuller et al, 1989; Peters et al, 2000). However, intermediate-risk factors such as LVSI, large tumour size, and deep stromal invasion (DSI) do not significantly increase the recurrence rate alone, but when combined, the risk of recurrence is increased to $15-20 \%$, which is similar to that of high-risk factors (Delgado et al, 1989, 1990; Sedlis et al, 1999; Van de Putte et al, 2005; Ryu et al, 2011).

Because selecting a criterion for a risk group involves choosing a specific point in the risk spectrum, researchers or physicians can use any criterion according to the purpose of the specific study or treatment strategy (e.g., a high-risk group for relatively toxic therapeutic agents and a low-risk group for relatively non-toxic agents) (Delgado et al, 1989, 1990; Sedlis et al, 1999; Van de Putte et al, 2005; Ryu et al, 2011).

According to the 'Classic criteria', the intermediate-risk group includes patients with any two of the following three intermediaterisk factors: a) tumour size $\geqslant 2 \mathrm{~cm}$, b) LVSI, and c) stromal invasion of more than one-third of the cervical wall (Delgado et al, 1989; Samlal et al, 1997; Van de Putte et al, 2005; Ryu et al, 2011). Despite their simplicity and convenience, the Classic criteria provide low specificity and they are associated with a recurrence rate of 5-8\%; therefore, many researchers and physicians are reluctant to apply these criteria to sophisticated or toxic therapeutic agents in this patient group (Samlal et al, 1997; Ryu et al, 2011; Rogers et al, 2012).

In contrast, based on a prospective study, the Gynecologic Oncology Group (GOG) defined the intermediate-risk group using various combinations of three factors (LVSI, DSI, and tumour size). This approach resulted in the following 'GOG criteria': a) positive capillary-LSVI, deep and middle third penetration, and clinical tumour size $\geqslant 2 \mathrm{~cm} ; b$ ) superficial third penetration and clinical tumour size $\geqslant 5 \mathrm{~cm}$; and $c$ ) negative capillary-lymphatic space involvement with middle or deep third penetration and clinical tumour size $\geqslant 4 \mathrm{~cm}$ (Delgado et al, 1989, 1990; Sedlis et al, 1999). However, despite their complexity, the sensitivity of these criteria is low, as almost half of the recurrences occur in patients who do not meet the GOG criteria (Ryu et al, 2011). Furthermore, based on a previous study of patients with cervical cancer with only squamous cell histology, the prognostic significance of adenocarcinoma histology, which accounts for $\sim 25 \%$ of all cervical cancers, was excluded from the GOG criteria (Delgado et al, 1989, 1990; Sedlis et al, 1999). Considering that the incidence of adenocarcinoma histology in cervical cancer was recently reported to be as high as $32 \%$, the significance of adenocarcinoma histology as a prognostic variable should be thoroughly investigated (Adegoke et al, 2012).

In this study, we sought to determine a new criterion to define the intermediate-risk group of patients with cervical cancer exhibiting any intermediate-risk factor after radical hysterectomy.

\section{MATERIALS AND METHODS}

Patients. From 1998 to 2008, 2158 patients with pathologically proven International Federation of Gynecology and Obstetrics (FIGO) stage IB-IIA cervical cancer and any intermediate-risk factor after radical hysterectomy were enrolled from 15 Korean Gynecologic Oncology Group (KGOG)-affiliated institutes.

Cervical cancer was staged according to the FIGO staging system using physical examination, chest radiography, intravenous pyelography, and abdominopelvic computed tomography (CT) or magnetic resonance imaging (MRI) findings. Cystoscopy and colonoscopy were performed if there was a suspicion of bladder or rectal involvement. Tumour size was determined by clinical palpation, inspection, or measurement of the largest diameter of the tumour using imaging modalities such as CT or MRI.

The operational record of abdominal or laparoscopic radical hysterectomy with bilateral pelvic lymphadenectomy, with or without para-aortic lymphadenectomy, was reviewed to determine the appropriateness of the surgical procedure. Pathological reports including the description of histology, DSI, LVSI, number of lymph nodes and positive nodes, parametrial tumour involvement, and tumour invasion of the resection margin were reported and reviewed. DSI was measured in reference to the fractional thickness of the cervix, which was divided into thirds. LVSI was recorded when tumour cells were identified microscopically within endothelium-lined spaces.

Patients who received neoadjuvant chemotherapy before surgery, those with a history of previous radiation therapy, those with cervical cancer found incidentally after simple hysterectomy, and those with metastatic cervical cancers were excluded.

Adjuvant treatment. Adjuvant therapy was determined by the physicians according to the guidelines for cervical cancer treatment at each institute; the treatment options included no further treatment, radiation alone, radiation therapy with chemotherapy, and chemotherapy alone. In brief, adjuvant radiation therapy was started within 4-6 weeks after surgery using a conventional fourfield technique. The radiation dose ranged from $40 \mathrm{~Gy}$ in 23 fractions to $50.4 \mathrm{~Gy}$ in 28 fractions (daily fractions of $1.8-2.0 \mathrm{~Gy}$ (5 fractions per week) over 4.5-6 weeks). The cisplatin-based concurrent chemotherapy regimens consisted of weekly cisplatin $\left(40 \mathrm{mg} / \mathrm{m}^{2}\right)$ for six cycles or FP $\left(500 \mathrm{mg} / \mathrm{m}^{2} 5\right.$-fluorouracil (FU) +50 $\mathrm{mg} / \mathrm{m}^{2}$ cisplatin) every 3 weeks for three cycles. The systemic chemotherapy regimens consisted of CP $\left(500 \mathrm{mg} / \mathrm{m}^{2}\right.$ cyclophosphamide $+50 \mathrm{mg} / \mathrm{m}^{2}$ cisplatin), FP $\left(500 \mathrm{mg} / \mathrm{m}^{2} 5-\mathrm{FU}+50 \mathrm{mg} / \mathrm{m}^{2}\right.$ cisplatin), or TP $\left(135 \mathrm{mg} / \mathrm{m}^{2}\right.$ paclitaxel $+75 \mathrm{mg} / \mathrm{m}^{2}$ cisplatin).

Follow-up. At follow-up visits, patients received a physical examination, Pap smear, and squamous cell carcinoma antigen monitoring every 3 months for 2 years and then every 6 months for the next 3 years. Chest radiography and imaging modalities such as $\mathrm{CT}$, positron emission tomography (PET), or PET/CT were performed every 6 months for the first 2 years and then annually for the next 3 years.

Disease recurrence was defined as the histological presence of tumour cells by biopsy or fine-needle aspiration cytology, any lymph node $>1 \mathrm{~cm}$ along the short axis diameter on the CT scan, or an increase in tumour size $>30 \%$ on the follow-up CT scan.

All medical records were collected according to the KGOG 1021 protocol and case report forms approved by each institutional review board, which waived the requirement for informed consent.

\section{Statistics}

Development group and validation group. A total of 2158 patients were included in this analysis. These subjects were randomly divided into development and validation groups at a 
3:1 allocation ratio. Models were developed using the development group and validated using the validation group.

Selection of prognostic variables. The association between clinicopathologic parameters was examined using the $\chi^{2}$-test with the Yates continuity correction for $2 \times 2$ tables, Fisher's exact test, and the Pearson correlation test, as appropriate. Disease-free survival was estimated using the Kaplan-Meier method. Survival curves were compared using the log-rank test. Trend tests were performed for ordinal scale categorical variables. The Cox proportional hazards regression model was used to estimate the hazard ratio of risk factors.

To develop prediction models for recurrence, univariate and multivariate Cox regression models were employed. FIGO stage, histology, tumour size, DSI, LVSI, and adjuvant treatment were considered in the analyses.

Tumour size was categorised as $2,3,4$, or $5 \mathrm{~cm}$, and DSI variables were stratified using 2 cutoff values (middle third or more and outer third).

Abbreviations and development of models. To simplify the name of each factor, we used the following abbreviations: ADE, adenocarcinoma or adenosquamous carcinoma histology; 2CM, tumour size $\geqslant 2 \mathrm{~cm} ; 3 \mathrm{CM}$, tumour size $\geqslant 3 \mathrm{~cm} ; 4 \mathrm{CM}$, tumour size $\geqslant 4 \mathrm{~cm} ; 5 \mathrm{CM}$, tumour size $\geqslant 5 \mathrm{~cm}$; MID, stromal invasion $\geqslant$ the middle third of the cervical wall; and OUT, stromal invasion of the outer third of the cervical wall.

For the selection of variables for the models, we validated the statistical significance of all possible combinations of factors, including two-factor models, any two factors among three-factor models, and any two or three factors among four-factor models.

As the four-factor model performed better than the two- and three-factor models, we only presented the results of the fourfactor models, which we termed Models 1-9.

Evaluation of the performance of the models. To evaluate the performance of the models with respect to their discrimination ability, two statistics were used: chi-square values from the logrank test and C-statistics from the receiver operating characteristic (ROC) analysis.

All analyses were performed using SAS v.9.0 and SPSS v.18.

\section{RESULTS}

Demographic characteristics. The median age of the participants was 49.3 years (range, 19.7-83 years). Stage IB1 disease $(76.7 \%)$ was the most common, followed by stage IB2 (11.7\%) and stage IIA (11.5\%) disease. Squamous cell carcinoma was the most common histologic cell type $(76.2 \%)$, followed by adenocarcinoma (16.7\%) and adenosquamous carcinoma (4.8\%; Table 1$)$.

The median tumour size was $2.8 \mathrm{~cm}$ (range, $1-7 \mathrm{~cm}$ ), and the distribution of the extent of DSI was even (inner third, 36.6\%; middle third, 29.1\%; and outer third, 34.3\%). Positive LVSI was found in $31.2 \%$ of the patients (Table 1 ).

The development and validation groups did not differ significantly in terms of age, stage, tumour size, or distribution of intermediate-risk factors (Table 1).

Two-thirds of patients met the Classic criteria (63.8\%), whereas $36.1 \%$ of patients met the GOG criteria. More than $70 \%$ of patients were observed without any adjuvant treatment, but $26.2 \%$ of patients required treatment, which primarily consisted of chemoradiation or radiation only $(20.4 \%)$ and systemic chemotherapy (5.8\%; Table 1).

The development and validation groups did not differ significantly with regard to risk factor distribution, and $64 \%$ and
Table 1. Demographic characteristics

\begin{tabular}{|l|c|c|c|}
\cline { 2 - 3 } \multicolumn{1}{c|}{} & \multicolumn{2}{c|}{ Number (\%) or median (range) } \\
\cline { 2 - 3 } & $\begin{array}{c}\text { Development } \\
\text { group }\end{array}$ & $\begin{array}{c}\text { Validation } \\
\text { group }\end{array}$ & \multirow{2}{*}{ P-value } \\
\hline No. of patients & 1620 & 538 & \\
\hline Age (years) & $49.3(19.7-83.0)$ & $48.7(26.9-78.0)$ & \\
\hline Stage & $1243(76.7)$ & $416(77.3)$ & ns \\
\hline IB1 & $190(11.7)$ & $50(9.3)$ & \\
IB2 & $187(11.5)$ & $72(13.4)$ & \\
IIA & \multicolumn{3}{|c|}{}
\end{tabular}

Histology

\begin{tabular}{|l|c|c|c|}
\hline SCC & $1234(76.2)$ & $403(74.9)$ & $\mathrm{ns}$ \\
Adeno & $271(16.7)$ & $90(16.7)$ & \\
Adenosquamous & $78(4.8)$ & $37(6.9)$ & \\
Other & $37(2.3)$ & $8(1.5)$ & \\
\hline Tumour size $(\mathrm{cm})$ & $2.8(1.0-7.0)$ & $2.7(1.0-7.0)$ & $\mathrm{ns}$ \\
\hline
\end{tabular}

\section{DSI}

\begin{tabular}{|l|c|c|c|}
\hline Inner 1/3 & $593(36.6)$ & $183(34.0)$ & ns \\
Middle $1 / 3$ & $472(29.1)$ & $171(31.8)$ & \\
Outer $1 / 3$ & $555(34.3)$ & $184(34.2)$ & \\
\hline LVSI & $505(31.2)$ & $166(30.9)$ & ns \\
& $1115(68.8)$ & $372(69.1)$ &
\end{tabular}

Intermediate-risk grouping

\begin{tabular}{|l|c|c|c|}
\hline Classic criteria (+) & $1034(63.8)$ & $344(63.9)$ & ns \\
GOG criteria (+) & $585(36.1)$ & $190(35.3)$ & \\
\hline
\end{tabular}

\section{Adjuvant treatment}

\begin{tabular}{|c|c|c|c|}
\hline $\begin{array}{l}\text { Yes } \\
\text { RT or CRT } \\
\text { Chemotherapy } \\
\text { No }\end{array}$ & $\begin{array}{c}425(26.2) \\
331(20.4) \\
94(5.8) \\
1195(73.8)\end{array}$ & $\begin{array}{c}143(26.6) \\
109(20.3) \\
34(6.3) \\
395(73.4)\end{array}$ & ns \\
\hline Follow-up duration (months) & $64.0(0.2-234.0)$ & $64.6(0.2-231.0)$ & ns \\
\hline $\begin{array}{l}\text { Recurrence } \\
\text { Classic criteria }(+) \\
\text { GOG criteria }(+)\end{array}$ & $\begin{array}{c}131 / 1620(8.1) \\
103 / 1034(10.0) \\
71 / 585(12.1)\end{array}$ & $\begin{array}{l}44 / 538(8.2) \\
30 / 344(8.7) \\
18 / 190(9.5)\end{array}$ & ns \\
\hline \multicolumn{4}{|l|}{ Status } \\
\hline $\begin{array}{l}\text { Died } \\
\text { Alive }\end{array}$ & $\begin{array}{c}78(4.8) \\
1533(94.6)\end{array}$ & $\begin{array}{c}27(5.0) \\
510(94.8)\end{array}$ & ns \\
\hline
\end{tabular}

Abbreviations: $C R T=$ concurrent chemoradiation; $D S I=$ deep stromal invasion; $G O G=$ Gynecologic Oncology Group; LVSI = lymphovascular space involvement; ns = not significant; $\mathrm{RT}=$ radiation therapy; $\mathrm{SCC}=$ squamous cell carcinoma

$35 \%$ of patients met the Classic and GOG criteria, respectively (Table 1).

After a median follow-up time of 64.2 months, 131 recurrences (8.1\%) were noted, and 78 patients died of the disease $(4.8 \%)$ in the development group; these values were not significantly different from those of the validation group (recurrence rate, $8.2 \%$ and death rate, $5 \%$; Table 1 ).

Pattern of recurrence. There were 175 recurrences (8.1\%) among the 2158 patients. There was no statistically significant difference in the number of recurrences between the no further treatment group $(8.1 \%)$ and those who received radiation therapy or 
chemoradiation (8.2\%). However, the recurrence rate was higher in patients who received adjuvant chemotherapy $(19.5 \%$, $P<0.05$; Table 2). There was no statistically significant difference in the number of recurrent sites among the three groups (Table 2).

Selection of models for discriminating the intermediate-risk group. FIGO stage, histology, tumour size, DSI, LVSI, and adjuvant treatment were significantly associated with disease-free survival in the univariate analysis, but FIGO stage and adjuvant treatment were not significantly associated with disease-free survival in the multivariate analysis (Table 3).

After excluding FIGO stage and adjuvant treatment as prognostic variables, nine models were established via various combinations of four variables (histology, tumour size, DSI, and LVSI) that exhibited statistical significance in multivariate analysis, and their significance was compared with the Classic and GOG criteria (Table 4).

Among the nine models, Model 2 (any two factors among 3CM, OUT, LVSI, and ADE) exhibited the highest chi-square score (40.48) in the log-rank test, followed by Model 4 (any three factors among 2CM, OUT, LVSI, and ADE; chi-square value, 39.33) and Model 5 (any three factors among 3CM, OUT, LVSI, and ADE; chi-square value, 36.47; Table 4). In addition to Models 2, 4, and 5, the other any two- or any three-factor models displayed higher chisquare scores than the Classic and GOG criteria (14.95 and 25.55, respectively).
All of the analyses were duplicated in the no further treatment only group, which did not receive any adjuvant treatment, and the highest chi-square value was observed in Model 2 (Table 4).

Performance analysis of models. Performance analysis for predicting recurrence among the 11 models, including the Classic and GOG criteria, revealed that Model 2 displayed the best performance among the various models ( $C$-value, 0.634 ), as confirmed in a separate analysis in the validation group ( $C$-value, 0.686; Table 5).

Receiver operating characteristic curve analysis also confirmed that Model 2 performed the best among the four-factor models, as well as markedly better than the Classic and GOG criteria (Figure 1).

\section{DISCUSSION}

This study demonstrated that our 'four-factor model' including any two of four intermediate-risk factors (3CM, LVSI, OUT, and ADE) could predict recurrence and survival more effectively than other criteria in patients with intermediate-risk factors after radical hysterectomy.

According to the Classic criteria, the intermediate-risk group is defined by the presence of any two factors among three variables (2CM, MID, and LVSI) (Delgado et al, 1989; Samlal et al, 1997; Van de Putte et al, 2005; Ryu et al, 2011). Despite their simplicity and convenience, the Classic criteria define the intermediate-risk

\section{Table 2. Pattern of recurrence}

\begin{tabular}{|c|c|c|c|c|c|}
\hline & No. (\%) & No further treatment (\%) & RT or CRT (\%) & Chemotherapy (\%) & $P$-value \\
\hline No. of patients & 2158 & 1718 & 440 & 128 & \\
\hline No. of recurrence & $175(8.1)$ & $139(8.1)$ & $36(8.2)$ & $25(19.5)$ & 0.0002 \\
\hline \multicolumn{6}{|c|}{ Site of recurrence } \\
\hline $\begin{array}{l}\text { Local } \\
\text { Distant } \\
\text { Mixed }\end{array}$ & $\begin{array}{l}66(3.1) \\
85(3.9) \\
24(1.1)\end{array}$ & $\begin{array}{l}56(3.3) \\
65(3.8) \\
18(1.0)\end{array}$ & $\begin{array}{c}10(2.3) \\
20(4.5) \\
6(1.4)\end{array}$ & $\begin{array}{c}8(6.3) \\
10(7.8) \\
7(5.5) \\
\end{array}$ & ns \\
\hline
\end{tabular}

Table 3. Univariate and multivariate analysis of individual variables for disease-free survival

\begin{tabular}{|c|c|c|c|c|c|c|c|}
\hline \multirow[b]{2}{*}{ Risk factors } & \multirow[b]{2}{*}{ Abbreviation } & \multicolumn{3}{|c|}{ Univariate analysis } & \multicolumn{3}{|c|}{ Multivariate analysis } \\
\hline & & HR & $95 \% \mathrm{Cl}$ & $P$-value & HR & $95 \% \mathrm{Cl}$ & $P$-value \\
\hline FIGO stage & ST2 & 1.900 & $1.229-2.937$ & 0.004 & 1.482 & $0.940-2.339$ & 0.091 \\
\hline Adeno or adenosquamous & $\mathrm{ADE}$ & 1.469 & $1.011-2.134$ & 0.043 & 1.743 & $1.194-2.545$ & 0.004 \\
\hline Tumour size $\geqslant 2 \mathrm{~cm}$ & $2 \mathrm{CM}$ & 4.883 & $1.997-11.938$ & 0.001 & 4.588 & $1.871-11.248$ & 0.001 \\
\hline Tumour size $\geqslant 3 \mathrm{~cm}$ & $3 \mathrm{CM}$ & 2.756 & $1.815-4.184$ & 0.000 & 2.512 & $1.645-3.835$ & 0.000 \\
\hline Tumour size $\geqslant 4 \mathrm{~cm}$ & $4 \mathrm{CM}$ & 1.833 & $1.289-2.606$ & 0.001 & 1.481 & $1.024-2.142$ & 0.037 \\
\hline Tumour size $\geqslant 5 \mathrm{~cm}$ & $5 \mathrm{CM}$ & 2.038 & $1.319-3.151$ & 0.001 & 1.537 & $0.978-2.415$ & 0.062 \\
\hline Stromal invasion $\geqslant$ middle $1 / 3$ of cervical wall & MID & 1.845 & $1.246-2.734$ & 0.002 & 1.303 & $0.861-1.973$ & 0.211 \\
\hline Stromal invasion $\geqslant$ outer $1 / 3$ of cervical wall & OUT & 2.020 & $1.435-2.843$ & 0.000 & 1.566 & $1.091-2.247$ & 0.015 \\
\hline LVSI positive & LVSI & 2.015 & $1.426-2.847$ & 0.000 & 1.845 & $1.265-2.690$ & 0.001 \\
\hline Adjuvant treatment & ADJ & 1.957 & $1.355-2.827$ & 0.000 & 1.372 & $0.910-2.069$ & 0.131 \\
\hline
\end{tabular}




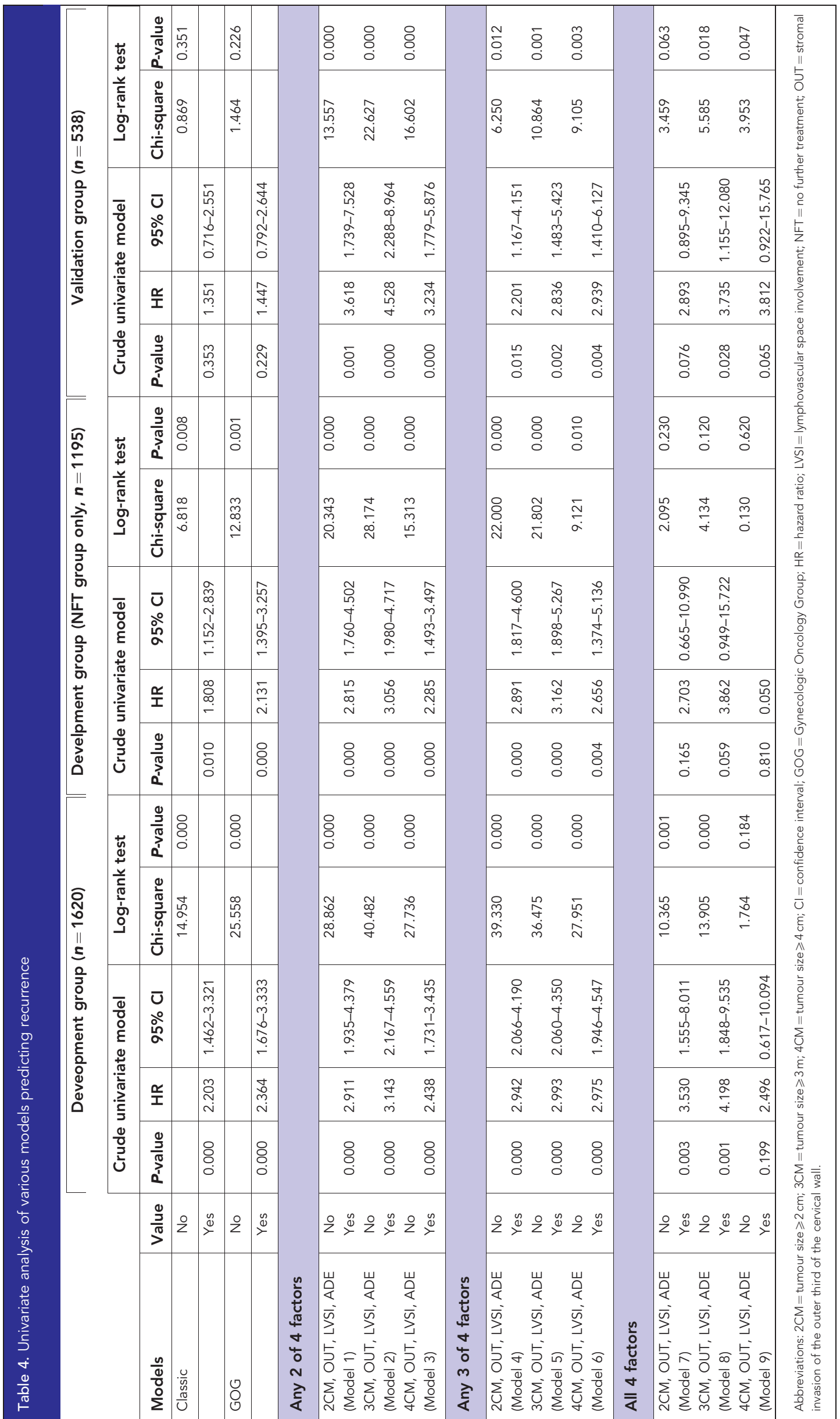


Table 5. C-value of each model for predicting recurrence

Development group $(n=1620)$

Validation group $(n=538)$

\begin{tabular}{|l|c|c|c|c|c|c|}
\hline Model & C-value $^{\mathrm{a}}$ & \multicolumn{2}{|c|}{$95 \% \mathrm{Cl}$} & C-value & \multicolumn{2}{|c|}{$95 \% \mathrm{Cl}$} \\
\hline Classic & 0.581 & 0.542 & 0.619 & 0.540 & 0.469 & 0.611 \\
\hline GOG & 0.613 & 0.567 & 0.659 & 0.555 & 0.475 & 0.634 \\
\hline Model 1 & 0.612 & 0.572 & 0.651 & 0.644 & 0.582 & 0.705 \\
\hline Model 2 & 0.634 & 0.591 & 0.677 & 0.686 & 0.620 & 0.752 \\
\hline Model 3 & 0.605 & 0.558 & 0.651 & 0.650 & 0.573 & 0.728 \\
\hline Model 4 & 0.613 & 0.566 & 0.659 & 0.658 & 0.580 & 0.736 \\
\hline Model 5 & 0.603 & 0.557 & 0.648 & 0.564 & 0.491 & 0.638 \\
\hline Model 6 & 0.584 & 0.542 & 0.627 & 0.586 & 0.513 & 0.659 \\
\hline Model 7 & 0.562 & 0.525 & 0.600 & 0.561 & 0.495 & 0.627 \\
\hline Model 8 & 0.525 & 0.498 & 0.553 & 0.567 & 0.504 & 0.629 \\
\hline Model 9 & 0.509 & 0.494 & 0.524 & 0.501 & 0.477 & 0.526 \\
\hline & & & & & &
\end{tabular}

Abbreviations: $\mathrm{Cl}=$ confidence interval; $\mathrm{GOG}=$ Gynecologic Oncology Group

a Disease-free survival, $t=60$ months.

group rather liberally, resulting in a relatively low specificity for predicting recurrence and survival (Ryu et al, 2011), which decreases the general performance of the criteria (Table 4). In contrast, the GOG criteria define the intermediate-risk group more strictly than the Classic criteria, as they include various combinations of the three factors (LVSI, DSI, and tumour size); however, these criteria are too complex and inconvenient, and they demonstrate low sensitivity and are poor predictors of recurrence and survival (Table 4) (Delgado et al, 1989; Sedlis et al, 1999; Ryu et al, 2011).

Our results indicate that the 'four-factor model,' which defines the intermediate-risk group according to the presence of any two of four risk variables (tumour size, DSI, LVSI, and histology), is a better predictor of recurrence and survival compared with previous criteria. We postulate that this difference in performance arises primarily from the inclusion of histologic cell type as an intermediaterisk factor, which was ignored in previous studies (Delgado et al, 1989, 1990; Sedlis et al, 1999; Van de Putte et al, 2005).

In general, adenocarcinomas of the cervix are considered to be more difficult to detect because they are detected at a more advanced stage and they metastasise earlier, resulting in a poorer prognosis, than similar-stage squamous cell cervical cancer tumours (Eifel et al, 1995; Farley et al, 2003; Park et al, 2010b; Lee et al, 2011; Rudtanasudjatum et al, 2011; Huang et al, 2012; Mabuchi et al, 2012). Adenocarcinomas of the cervix are also more resistant to radiation therapy due to the presence of highly cycling cells, including a high proportion of residual radioresistant cells, compared with squamous cell cancer (Eifel et al, 1995; Shibata et al, 2009). However, in the intermediate-risk group of patients with cervical cancer who have a favourable prognosis, the statistical significance of histologic type has been ignored in many reports and clinical trials (Delgado et al, 1989, 1990; Sedlis et al, 1999; Farley et al, 2003; Van de Putte et al, 2005; Rudtanasudjatum et al, 2011). Our results demonstrated that histologic cell type has significant prognostic value, and if included as an intermediate-risk factor, it could dramatically improve the performance of criteria for defining the intermediate-risk group among patients with early cervical cancer after radical hysterectomy.

Tumour size is strongly correlated with recurrence and survival in cervical cancer after radical hysterectomy (Delgado et al, 1989;
Bansal et al, 2009; Turan et al, 2010; Garg et al, 2011; Chang et al, 2012) (Tables 3-4). In general, 2CM is correlated with an increased risk of recurrence (Delgado et al, 1989; Chang et al, 2012), but our study and others indicate that $3 \mathrm{CM}$ is better correlated with recurrence (Table 5).

The measurement of tumour size in cervical cancer is frequently debated. Currently, several methods have been proposed for measuring tumour size in cervical cancer, including clinical palpation, measurement of the longest diameter on imaging modalities, the colposcopic tumour size, and measurement of the diameter of the pathologic specimen. However, each of these methods has limitations such as subjectivity, tumour shrinkage, and difficulty in measuring endocervical cancers. Currently, despite limited subjectivity, clinical tumour size determined by palpation under general anaesthesia is generally accepted as a standard method of tumour measurement in cervical cancer. In this study, because tumour size was measured primarily using imaging modalities such as MRI, we suggest that our results may be less controversial than those presented in previous studies.

Despite the retrospective nature of this study, it is the largest study to date, particularly regarding the number of patients with adenocarcinoma or adenosquamous carcinoma histology, which increases the credibility of our results. The largest published study to date included 732 cases of stage IB cervical cancer with squamous histology only (Delgado et al, 1989). The total number of patients in our study exceeded 2000, including 400 cases with adenocarcinoma or adenosquamous carcinoma histology, which was sufficient to assess the significance of the histologic cell type of cervical cancer.

This study also involved 15 institutes and encompassed a 10 -year study period. Despite the possible diversity in pathologist review methods and the modality of treatment among the institutes, our results more accurately reflect the real-world clinical situation than short-term prospective studies. We do not believe that the pathologist's decision to determine LVSI, DSI, and histologic cell type is difficult, and the treatment of cervical cancer has changed significantly since the introduction of cisplatin-based chemoradiation in the 1990s (Rogers et al, 2012). Therefore, we believe that the confounding effect of varying pathologic review methods at each institute and changes in treatment during the study period are negligible.

In this study, over a median follow-up period of more than 60 months, the overall recurrence rate was $8.2 \%$, which is lower than that reported in previous studies (Delgado et al, 1989; Van de Putte et al, 2005). The more general application of adjuvant treatment to the relatively higher risk group may have reduced the recurrence rate; however, despite adjuvant treatment, the recurrence rate did not decrease below the level of the non-adjuvant treatment group (Table 2). Moreover, although there was no statistical significance, a slight difference was observed in the recurrence pattern in the CT or CRT groups, which demonstrated lower local recurrence and higher distant metastasis rates, suggesting that adjuvant RT or CRT is a useful local control modality but does not prevent distant metastasis. Furthermore, the adjuvant chemotherapy group showed the highest recurrence rate $(19.5 \%)$, strongly suggesting that adjuvant chemotherapy may not serve as an effective adjuvant treatment modality. However, because this study was not designed to investigate the role of adjuvant treatment, further studies are needed to confirm this finding. In addition, adjuvant treatment may influence recurrence and survival; however, the statistical significance of our models was not affected by the inclusion of patients who received adjuvant therapy (Table 4). Regarding the homogeneity of the study population, excluding the adjuvant treatment group seemed to be a more reasonable approach than including it, but this also biased our results by removing the relatively higher risk group of patients. Table 4 presents the analysis in both ways, excluding or including the adjuvant 


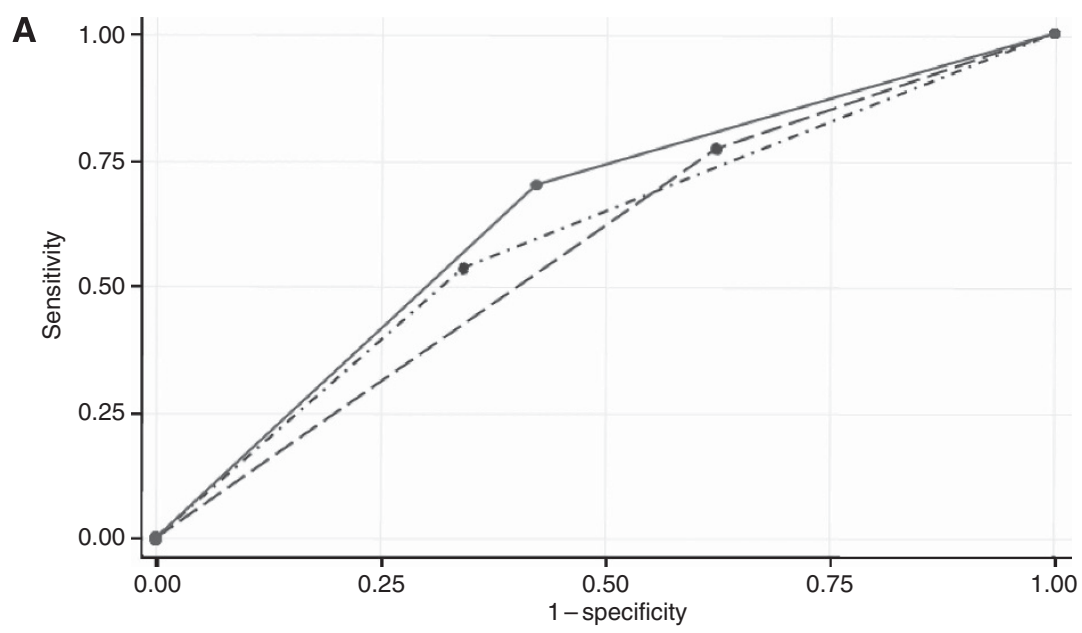

\begin{tabular}{|c|c|c|c|c|c|c|c|}
\hline \multirow{2}{*}{$\begin{array}{l}\text { Test } \\
\text { Model }\end{array}$} & \multicolumn{3}{|c|}{$\begin{array}{l}-\cdot-\bullet-\cdot-\text { GOG ROC area: } 0.5967 \\
\longrightarrow-\text { Model2 ROC area: } 0.6384\end{array}$} & \multicolumn{4}{|c|}{$-\rightarrow--$ Classic ROC area: 0.5739} \\
\hline & Obs & $\mathrm{ROC}$ area & s.e. & \multicolumn{2}{|c|}{$95 \% \mathrm{Cl}$} & Chi-square & $P$-value \\
\hline GOG & 1568 & 0.5967 & 0.0231 & 0.551 & 0.642 & & \\
\hline Classic & 1568 & 0.5739 & 0.0198 & 0.535 & 0.613 & 10.7 & 0.0048 \\
\hline Model2 & 1568 & 0.6384 & 0.0214 & 0.596 & 0.680 & & \\
\hline
\end{tabular}

Ho: area $(\mathrm{GOG})=$ area $($ Classic $)=$ area $($ Model 2$)$

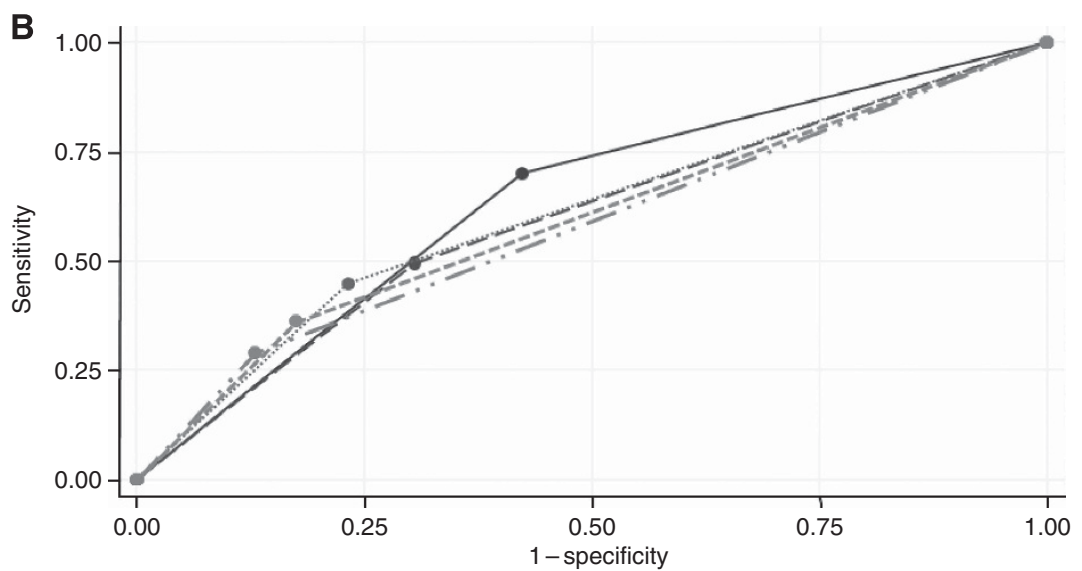

\begin{tabular}{|c|c|c|c|c|c|c|c|}
\hline Test & \multicolumn{3}{|c|}{ 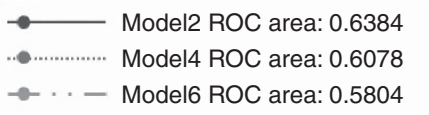 } & \multicolumn{4}{|c|}{$\begin{array}{l}\longrightarrow--- \text { Model3 ROC area: } 0.595 \\
----\cdot \text { Model5 ROC area: } 0.5933\end{array}$} \\
\hline Model & Obs & $\mathrm{ROC}$ area & s.e. & \multicolumn{2}{|c|}{$95 \% \mathrm{Cl}$} & Chi-square & $P$-value \\
\hline Model 2 & 1568 & 0.6384 & 0.0214 & 0.596 & 0.680 & & \\
\hline Model 3 & 1568 & 0.595 & 0.0231 & 0.550 & 0.640 & & \\
\hline Model 4 & 1568 & 0.6078 & 0.0228 & 0.563 & 0.653 & 10.9 & 0.0277 \\
\hline Model 5 & 1568 & 0.5933 & 0.0220 & 0.550 & 0.636 & & \\
\hline Model 6 & 1568 & 0.5804 & 0.0207 & 0.540 & 0.621 & & \\
\hline
\end{tabular}

Ho: area $($ Model 2$)=$ area $($ Model 3$)=$ area $($ Model 4$)=$ area $($ Model 5) $=$ area $($ Model 6$)$

Figure 1. The ROC curve analysis for recurrence confirmed that Model 2 performed better than the Classic and GOG criteria (A) and other models (B). Abbreviations: $\mathrm{Cl}=$ confidence interval; $\mathrm{HO}=$ null hypothesis; $\mathrm{Obs}=$ observed; $\mathrm{ROC}=$ receiver operating characteristic.

treatment group, which ultimately produced the same results. In conclusion, our study identified a 'four-factor model' that used the presence of any two of four risk variables (3CM, OUT, LVSI, and $\mathrm{ADE}$ ) to define the intermediate-risk group. This model may predict recurrence and poor survival more effectively than previous criteria in patients with cervical cancer and the presence of any intermediate-risk factor after radical hysterectomy. Moreover, our model will be beneficial to researchers and physicians for selecting which group of patients should receive adjuvant treatment and for defining patient groups for clinical trials aimed at investigating more sophisticated treatment strategies.

\section{ACKNOWLEDGEMENTS}

We thank the following participating institutions: Korea Institute of Radiological \& Medical Sciences, University of Ulsan College of 
Medicine, Sungkyunkwan University School of Medicine, Busan Paik Hospital, Chonnam National University Medical School, Inje University College of Medicine, Keimyung University, National Cancer Center, Ajou University School of Medicine, Seoul National University Bundang Hospital, Seoul National University, Gachon University Gil Hospital, Inha University Hospital, Seoul Metropolitan Boramae Hospital, and Konkuk University Medical Center. This study was supported by a grant from the Korea Institute of Radiological and Medical Sciences (KIRAMS RTR: 50457-2012).

\section{CONFLICT OF INTEREST}

The authors declare no conflict of interest.

DISCLAIMER

The funding sources had no role in the design of the study; the collection, analysis, and interpretation of data; the writing of the report; or in the decision to submit the paper for publication.

\section{REFERENCES}

Adegoke O, Kulasingam S, Virnig B (2012) Cervical cancer trends in the United States: a 35-year population-based analysis. J Womens Health 21: 1031-1037.

Bansal N, Herzog TJ, Shaw RE, Burke WM, Deutsch I, Wright JD (2009) Primary therapy for early-stage cervical cancer: radical hysterectomy $v s$ radiation. Am J Obstet Gynecol 201: 485 e1-e9.

Chang SJ, Bristow RE, Ryu HS (2012) A model for prediction of parametrial involvement and feasibility of less radical resection of parametrium in patients with FIGO stage IB1 cervical cancer. Gynecol Oncol 126: 82-86.

Delgado G, Bundy BN, Fowler Jr WC, Stehman FB, Sevin B, Creasman WT, Major F, DiSaia P, Zaino R (1989) A prospective surgical pathological study of stage I squamous carcinoma of the cervix: a Gynecologic Oncology Group Study. Gynecol Oncol 35: 314-320.

Delgado G, Bundy B, Zaino R, Sevin BU, Creasman WT, Major F (1990) Prospective surgical-pathological study of disease-free interval in patients with stage IB squamous cell carcinoma of the cervix: a Gynecologic Oncology Group study. Gynecol Oncol 38: 352-357.

Eifel PJ, Burke TW, Morris M, Smith TL (1995) Adenocarcinoma as an independent risk factor for disease recurrence in patients with stage IB cervical carcinoma. Gynecol Oncol 59: 38-44.

Farley JH, Hickey KW, Carlson JW, Rose GS, Kost ER, Harrison TA (2003) Adenosquamous histology predicts a poor outcome for patients with advanced-stage, but not early-stage, cervical carcinoma. Cancer 97: 2196-2202.

Fuller AF, Elliott N, Kosloff C, Hoskins WJ, Lewis Jr JL (1989) Determinants of increased risk for recurrence in patients undergoing radical hysterectomy for stage IB and IIA carcinoma of the cervix. Gynecol Oncol 33: 34-39.

Garg G, Shah JP, Toy EP, Christensen C, Deppe G, Asaad R, Morris RT (2011) Stage IIA1 $v s$ stage IIA2 cervical cancer: does the new staging criteria predict survival? Int J Gynecol Cancer 21: 711-716.

Huang YT, Wang CC, Tsai CS, Lai CH, Chang TC, Chou HH, Lee SP, Hong JH (2012) Clinical behaviors and outcomes for adenocarcinoma or adenosquamous carcinoma of cervix treated by radical hysterectomy and adjuvant radiotherapy or chemoradiotherapy. Int J Radiat Oncol Biol Phys 84: $420-427$.

Lee YY, Choi CH, Kim TJ, Lee JW, Kim BG, Lee JH, Bae DS (2011) A comparison of pure adenocarcinoma and squamous cell carcinoma of the cervix after radical hysterectomy in stage IB-IIA. Gynecol Oncol 120: $439-443$.

Mabuchi S, Okazawa M, Matsuo K, Kawano M, Suzuki O, Miyatake T, Enomoto T, Kamiura S, Ogawa K, Kimura T (2012) Impact of histological subtype on survival of patients with surgically-treated stage IA2-IIB cervical cancer: adenocarcinoma vs squamous cell carcinoma. Gynecol Oncol 127: 114-120.

Park JY, Kim DY, Kim JH, Kim YM, Kim YT, Nam JH (2010a) Further stratification of risk groups in patients with lymph node metastasis after radical hysterectomy for early-stage cervical cancer. Gynecol Oncol 117: 53-58.

Park JY, Kim DY, Kim JH, Kim YM, Kim YT, Nam JH (2010b) Outcomes after radical hysterectomy in patients with early-stage adenocarcinoma of uterine cervix. Br J Cancer 102: 1692-1698.

Peters 3rd WA, Liu PY, Barrett RJ, Stock RJ, Monk BJ, Berek JS, Souhami L, Grigsby P, Gordon Jr W, Alberts DS (2000) Concurrent chemotherapy and pelvic radiation therapy compared with pelvic radiation therapy alone as adjuvant therapy after radical surgery in high-risk early-stage cancer of the cervix. J Clin Oncol 18: 1606-1613.

Rogers L, Siu SS, Luesley D, Bryant A, Dickinson HO (2012) Radiotherapy and chemoradiation after surgery for early cervical cancer. Cochrane Database Syst Rev(5): CD007583.

Rudtanasudjatum K, Charoenkwan K, Khunamornpong S, Siriaunkqul S (2011) Impact of histology on prognosis of patients with early-stage cervical cancer treated with radical surgery. Int J Gynaecol Obstet 115: 183-187.

Ryu SY, Park SI, Nam BH, Cho CK, Kim K, Kim BJ, Kim MH, Choi SC, Lee ED, Lee KH (2011) Is adjuvant chemoradiotherapy overtreatment in cervical cancer patients with intermediate risk factors? Int J Radiat Oncol Biol Phys 79: 794-799.

Samlal RA, van der Velden J, Ten Kate FJ, Schilthuis MS, Hart AA, Lammes FB (1997) Surgical pathologic factors that predict recurrence in stage IB and IIA cervical carcinoma patients with negative pelvic lymph nodes. Cancer 80: $1234-1240$.

Sedlis A, Bundy BN, Rotman MZ, Lentz SS, Muderspach LI, Zaino RJ (1999) A randomized trial of pelvic radiation therapy $v s$ no further therapy in selected patients with stage IB carcinoma of the cervix after radical hysterectomy and pelvic lymphadenectomy: a Gynecologic Oncology Group Study. Gynecol Oncol 73: 177-183.

Shibata K, Kajiyama H, Yamamoto E, Terauchi M, Ino K, Nomura S, Nawa A, Kawai M, Kikkawa F (2009) Effectiveness of preoperative concurrent chemoradiation therapy (CCRT) for locally advanced adenocarcinoma of cervix. Eur J Surg Oncol 35: 768-772.

Turan T, Yildirim BA, Tulunay G, Boran N, Kose MF (2010) Prognostic effect of different cut-off values $(20 \mathrm{~mm}, 30 \mathrm{~mm}$ and $40 \mathrm{~mm})$ for clinical tumour size in FIGO stage IB cervical cancer. Surg Oncol 19: 106-113.

Van de Putte G, Lie AK, Vach W, Baekelandt M, Kristensen GB (2005) Risk grouping in stage IB squamous cell cervical carcinoma. Gynecol Oncol 99: 106-112.

This work is published under the standard license to publish agreement. After 12 months the work will become freely available and the license terms will switch to a Creative Commons AttributionNonCommercial-Share Alike 3.0 Unported License. 